\title{
Improved SNR to detect the unknown characteristic frequency by $S R$
}

ISSN 1751-8822

Received on 30th January 2018

Revised 23rd April 2018

Accepted on 4th May 2018

E-First on 29th May 2018

doi: 10.1049/iet-smt.2018.0046

www.ietdl.org

\author{
Jingling Zhang ${ }^{1,2}$, Jianhua Yang ${ }^{1,2} \bowtie$, Houguang Liu'1,2, Dengji Zhou ${ }^{3,4}$ \\ ${ }^{1}$ School of Mechatronic Engineering, China University of Mining and Technology, Xuzhou 221116, People's Republic of China \\ 2 Jiangsu Key Laboratory of Mine Mechanical and Electrical Equipment, China University of Mining and Technology, Xuzhou 221116, People's \\ Republic of China \\ ${ }^{3}$ Key Laboratory of Power Machinery and Engineering, Shanghai Jiao Tong University, Shanghai 200240, People's Republic of China \\ ${ }^{4}$ Department of Mechanical Engineering, University of Michigan, Ann Arbor, MI 48109, USA \\ 凶-E-mail: jianhuayang@cumt.edu.cn
}

\begin{abstract}
Stochastic resonance (SR) is widely used in signal processing issues. The classic evaluation index of SR must know the characteristic frequency in prior. However, the accuracy frequency which needs to be detected is not known in advance. To solve this problem, the authors propose a new index, which calls improved signal-to-noise ratio (SNR) in adaptive SR. This new index is effective without knowing the accuracy characteristic frequency first. Meanwhile, the general scale transformation and random particle swarm optimisation algorithm are used to satisfy the conditions of SR and help to obtain the optimal system parameters. On the basis of this new index, the simulation and experimental bearing fault signals are both processed perfectly when compared with the classic SNR index. More importantly, it overcomes the drawbacks of the classic SNR index that the accuracy characteristic frequency must be known in advance. Therefore, these results indicate that new index has important practical values in signal processing issues.
\end{abstract}

\section{Introduction}

Bearing vibration signal is one kind of engineering signal that may contain different characteristic frequencies. However, rolling element bearings are vulnerable to the threats of mechanical failure under a harsh working environment. Moreover, these weak signals with bearing fault information become more difficult to be identified when they are buried by background noises. Therefore, recognising the characteristic fault from bearing vibration time series in noise background is a hot topic. Stochastic resonance (SR) which was first proposed by Benzi has become a popular method in signal processing issues [1]. Therefore, the SR method has significant applications in bearing fault diagnosis. This method is different from the traditional methods such as wavelet analysis [2, $3]$, neural network $[4,5]$ and empirical mode decomposition [6, 7]. The unique feature of SR is the noise energy transfers to the weak signal which contains feature information [8] rather than suppressing the noise in the raw signals. As a result, the proposed SR method is not only effective but also avoids the deterioration of useful bearing characteristic information. The traditional SR is excited by adjusting the noise intensity until the index reaches its maximum value. However, the noise is hard to change, especially to reduce, in real engineering environment. In this case, adaptive SR which fixes the noise and optimises the system parameters is used to adapt to the actual situation $[9,10]$. In terms of the adaptive SR, many optimisation algorithms [11-13] have been studied to get the optimal system parameters of adaptive SR. Herein, the random particle swarm optimisation algorithm [14-16] is used to optimise the system parameters. In most studies, signal-to-noise ratio (SNR) is the most common index in the SR method [17]. Although the SNR plays an important role in the SR method, it still has a fatal drawback that it should know the accuracy characteristic frequency in advance when the value of SNR is calculated. However, it is impossible to know the accuracy characteristic frequency in prior in most real situations. Hence, we propose a new index which is called as improved SNR (ISNR). In the calculation of the ISNR, we do not need to know the accuracy characteristic frequency first. The ISNR is obtained just needed to know the approximated characteristic frequency, which is received by the corresponding computational formula or according to engineering experience. Owing to the accuracy characteristic frequency has a little error with the approximated one, it can be successfully found in the range of the neighbourhood of the approximated value.

In consideration of the classic SR method only fitting for small parameters, Tan et al. [18, 19] and Leng et al. proposed a frequency-shifted and re-scaling SR method. Leng also puts forward the twice sampling SR to solve this limit [20]. Moreover, He et al. use multiscale noise tuning SR to realise the weak signal detection $[21,22]$. Among these methods, the multiscale noise tuning SR is a most widely used method. This method is usually realised by the normalised scale transformation and this transformation method may not achieve the optimal SR. Herein, we use the method of general scale transformation to solve this limitation of SR [23].

The rest of the contents of this paper are arranged as follows. The related theories of SR, the definition of ISNR and the optimisation algorithm are introduced in Section 2. In Sections 3 and 4 , the feasibility of ISNR is proved by the simulation signal and the experimental signal, respectively. Meanwhile, the adaptive SR with classic SNR is also used to make a comparison. Finally, the main conclusions are given in Section 5 .

\section{Theory and model}

In this section, we first introduce the theory of SR and its relevant calculation method. Then, the new model of ISNR is defined. Finally, the random particle swarm optimisation algorithm is briefly introduced. The specific processes of this method are revealed in Fig. 1.

\subsection{Theory of SR}

The model of SR subjected to external periodic driving force and noise is usually described by the following Langevin equation [24, 25]: 


$$
\left\{\begin{array}{l}
\frac{\mathrm{d} x}{\mathrm{~d} t}=-U^{\prime}(x)+s(t)+n(t) \\
\langle n(t)\rangle=0,\langle n(t), n(0)\rangle=2 D \delta(t)
\end{array},\right.
$$

where $s(t)$ represents the fault signal and $n(t)$ symbolises the Gaussian white noise. $D$ is the intensity of noise. $\delta(t)$ is the Dirac delta function.

As the typical model of SR, the bistable system is widely used. Its potential function is

$$
U(x)=-\frac{a}{2} x^{2}+\frac{b}{4} x^{4}
$$

In (2), $a$ and $b$ are the positive system parameters.

The following Langevin equation can be obtained by substituting (2) into (1):

$$
\frac{\mathrm{d} x}{\mathrm{~d} t}=a x-b x^{3}+s(t)+n(t)
$$

To realise the general scale transformation, some variables are introduced

$$
x(t)=z(\tau), \quad \tau=m t .
$$

Substituting (4) into (3) and letting $\mathrm{s}(t)=A \sin (2 \pi f t)$. Equation (3) can be rewritten as

$$
\left\{\begin{array}{l}
m \frac{\mathrm{d} z}{\mathrm{~d} \tau}=a z-b z^{3}+A \sin \left(2 \pi \frac{f}{m} \tau\right)+n\left(\frac{\tau}{m}\right) \\
n\left(\frac{\tau}{m}\right)=\sqrt{2 D m} \xi(\tau)
\end{array},\right.
$$

where $m$ is the time scale.

Equation (5) is simplified as

$$
\left\{\begin{array}{l}
\frac{\mathrm{d} z}{\mathrm{~d} \tau}=\frac{a}{m} z-\frac{b}{m} z^{3}+\frac{A}{m} \sin \left(2 \pi \frac{f}{m} \tau\right)+\sqrt{\frac{2 D}{m}} \xi(\tau), \\
\langle\xi(\tau)\rangle=0,\langle\xi(\tau), \xi(0)\rangle=\delta(\tau)
\end{array}\right.
$$

Letting $a_{1}=(a / m), b_{1}=(b / m)$. Equation (6) is the equivalent form of (3). In addition, the system parameters $a_{1}$ and $b_{1}$ are optimised by the random particle swarm optimisation algorithm after the general scale transformation. Then, the values of $a$ and $b$ are obtained by multiplying the time scale $m$. Herein, this type of equation can be solved by the fourth-order Runge-Kutta algorithm $[26,27]$.

\subsection{Model of ISNR}

As we know, the SNR refers to the ratio of signal energy and noise energy. To distinguish the definition of the classic SNR and the new index ISNR, we use $\mathrm{SNR}_{\mathrm{c}}$ and $\mathrm{SNR}_{\mathrm{I}}$ to represent them, respectively, in the calculation formula. The specific expression of classic SNR is

$$
\left\{\begin{array}{l}
\mathrm{SNR}_{\mathrm{c}}=10 \lg \frac{S\left(f_{\mathrm{r}}\right)}{N\left(f_{\mathrm{r}}\right)} \\
S\left(f_{\mathrm{r}}\right)=\left|X\left(k_{\mathrm{r}}\right)\right|^{2} \\
N\left(f_{\mathrm{r}}\right)=\sum_{i=k_{\mathrm{r}}-M}^{k_{\mathrm{r}}+M}|X(i)|^{2}-S\left(f_{\mathrm{r}}\right)
\end{array},\right.
$$

where $f_{\mathrm{r}}$ is the accuracy characteristic frequency of the input signal and $k_{\mathrm{r}}$ is the serial number of $f_{\mathrm{r}} \cdot X(\cdot)$ is the amplitude of the response and $X\left(k_{\mathrm{r}}\right)$ is the amplitude at $f_{\mathrm{r}}$. $S\left(f_{\mathrm{r}}\right)$ denotes the energy of input signal at the accuracy characteristic frequency. $N\left(f_{\mathrm{r}}\right)$ denotes the energy of noise around $f_{\mathrm{r}}$. It is calculated in the interval $f_{\mathrm{r}}-M \Delta f_{\mathrm{r}}$ and $f_{\mathrm{r}}+M \Delta f_{\mathrm{r}}$ and excludes $f_{\mathrm{r}}$, where $\Delta f_{\mathrm{r}}$ is the frequency step of the discrete amplitude spectrum. Therefore, the SNR here is the local

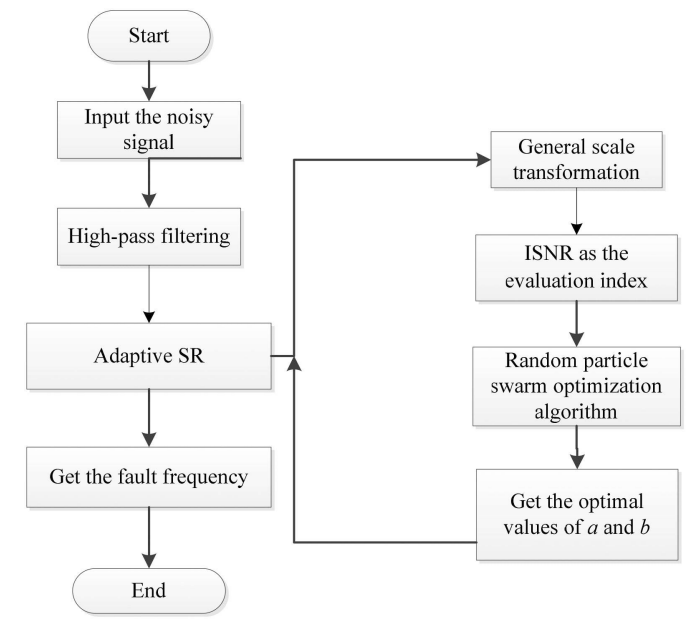

Fig. 1 Flowchart of the proposed method

SNR because of the choice of $M$. Herein, we should know the accuracy characteristic frequency in prior when we calculate the value of the classic SNR.

However, it is difficult to know the accuracy characteristic frequency in most practical cases. Therefore, it is meaningful to put forward this new index ISNR. The expression of ISNR is

$$
\left\{\begin{array}{l}
\mathrm{SNR}_{\mathrm{I}}=10 \lg \frac{S\left(f_{\mathrm{t}}\right)}{N\left(f_{\mathrm{t}}\right)} \\
S\left(f_{\mathrm{t}}\right)=\sum_{i=k_{\mathrm{t}}-l}^{k_{\mathrm{t}}+l}|X(i)|^{2} \\
N\left(f_{\mathrm{t}}\right)=\sum_{j=k_{\mathrm{t}}-M}^{k_{\mathrm{t}}+M}|X(j)|^{2}-S\left(f_{\mathrm{t}}\right)
\end{array}\right.
$$

where $f_{\mathrm{t}}$ is the theoretical fault frequency and $k_{\mathrm{t}}$ is the serial number of $f_{\mathrm{t}} . S\left(f_{\mathrm{t}}\right)$ represents the total energy which in the vicinity of $f_{\mathrm{t}}-l \Delta f_{\mathrm{t}}$ and $f_{\mathrm{t}}+l \Delta f_{\mathrm{t}}$ and it is centred on $f_{\mathrm{t}}$. The definition of $\Delta f_{\mathrm{t}}$ is similar to $\Delta f_{\mathrm{r}}$, but $\Delta f_{\mathrm{t}}$ is the result under the condition of calculating ISNR. $N\left(f_{\mathrm{t}}\right)$ denotes the energy of noise in a certain range and it excludes the $S\left(f_{t}\right)$. Its calculation method is the same as (7). In this case, we do not need to know the accuracy characteristic frequency in advance and we can obtain the theoretical fault frequency through the corresponding fault calculation formula. Then, the accuracy characteristic frequency can be easily found near the theoretical fault frequency. This is the unique advantage of this new index.

\subsection{Optimisation algorithm}

Herein, we use the random particle swarm optimisation algorithm to optimise the system parameters. The main steps of this algorithm are as follows:

Step 1: Set the initialisation condition: Determine the learning factors, the mean of the random weight, the number of iterations, the number of particles and the spatial dimension. The learning factors are generally set as 2 . The maximum mean and minimum mean of the random weight is set as 0.8 and 0.5 , respectively. The variance of the mean random weight is set as 0.2 . The number of iterations and particles are set as 40 and 50, respectively. Here, we optimise two system parameters, so the spatial dimension is set as 2.

Step 2: Initialise individual particles in the population: Randomly initialise the position and velocity of a particle here.

Step 3: Calculate the fitness of each particle and find the local optimal value and global optimal value. Herein, the fitness refers to the specific evaluation index and we use ISNR as the evaluation index in this paper. 

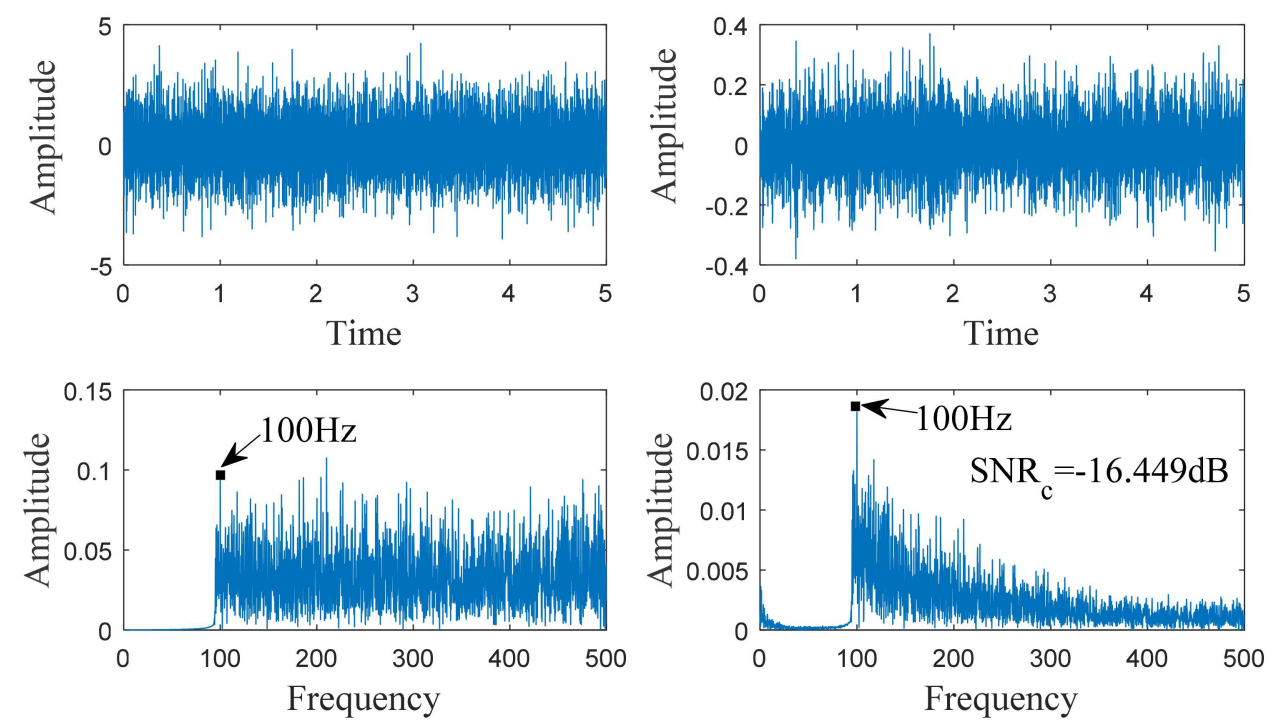

a

$b$

Fig. 2 Time-domain waveforms and frequency spectrums of the noisy signal before adaptive SR and after adaptive SR with the classic index SNR (a) Time-domain waveform and frequency spectrum of the noisy signal before adaptive SR, (b) Time-domain waveform and frequency spectrum of the noisy signal after adaptive SR with the classic index SNR. Optimal output with $a=0.516$ and $b=1459.125$

Step 4: Enter the main loop: First, update the position and velocity of the particle according to the calculation formula of the particle swarm optimisation algorithm. Second, recalculate the fitness of each particle and update the local optimal value and global optimal value. Then, judge whether the optimal value is between 0 and 2 . Finally, judge whether the maximum number of iterations has been reached. If not, continuing the loop. If reached, go to the next step. Step 5: Obtain the optimal system parameters.

By the random particle swarm optimisation algorithm, the optimal values of $a_{1}$ and $b_{1}$ are obtained at adaptive SR of a small parameter. Then, the optimal system parameters of $a$ and $b$ can be obtained according to time scale $m$.

\section{Numerical simulation}

In this section, we verify the effectiveness of this new index ISNR. Herein, we use a harmonic signal to simulate fault signal. The expression of this harmonic signal is

$$
x(t)=0.1 \cos (2 \pi \times 100 t) .
$$

To simulate the noise environment, we add the Gaussian white noise with the intensity of 1 into the harmonic signal. Herein, the sampling frequency is 1000 and the number of sampling points is 5000 . The passband frequency and stopband frequency of the highpass filter are set as 95 and $90 \mathrm{~Hz}$, respectively. The scale of time $m$ is set as 1000 , in general scale transformation. In the calculations of SNR and ISNR, $M$ is set as 500. Fig. $2 a$ shows the time-domain waveform and frequency spectrum of the noisy signal under the high-pass filter and before SR. From Fig. $2 a$, we find the fault frequency is submerged in the noise background and it is hardly to be found.

First, the classic index SNR is used in adaptive SR to process the noisy signal. The time-domain waveform and frequency spectrum of the noisy signal after adaptive SR is shown in Fig. $2 b$. As we see from Fig. $2 b$, the fault frequency appears clearly in the frequency spectrum and the adaptive SR performs well.

Then, we use the new index ISNR in adaptive SR and three ranges are selected to verify the validity of the index. The first case is the frequency range of $99-101 \mathrm{~Hz}$ and $l$ is set as 5 . The second case is the frequency range of $98-102 \mathrm{~Hz}$ and $l$ is set as 10 . The third case is the frequency range of $97-103 \mathrm{~Hz}$ and $l$ is set as 15 . Fig. 3 shows the time-domain waveforms and frequency spectrums about these three situations, respectively. Table 1 shows the values of the classic SNR and the new ISNR before and after adaptive SR. From Fig. 3 and Table 1, we find the fault frequency is easily identified after adaptive SR and the value of ISNR is improved greatly. Therefore, the adaptive SR with the new index ISNR has the same good effect as the classic index SNR.

To prove the new index ISNR is suitable for a small range of fault frequency changes in different noise intensities, Fig. 4 expresses the relationship between the amplitude of the fault frequency and bandwidth after adaptive SR. Here, bandwidth $\Delta f=l \times\left(f_{\mathrm{s}} / P\right) \times 2=l \times \Delta f_{\mathrm{t}} \times 2$. In this formula, $f_{\mathrm{s}}$ is the sampling frequency and $P$ is the sampling number. On account of the theoretical fault frequency is equal to the accurate fault frequency in the simulation signal, the case of $\Delta f=0$ is the adaptive SR with the classic index SNR. From Fig. 4, the amplitude of the fault frequency is basically unchanged in the search range no matter which value of $l$ at the situations of different noise intensities. More importantly, this new method has better robustness because the good effect of adaptive SR with ISNR is not influenced by noise intensities. Therefore, the accuracy characteristic frequency can be clearly observed when seeking it in the range of the neighbourhood of the theoretical fault frequency.

\section{Experimental verification}

In this section, we verify the effectiveness of this new index ISNR in adaptive SR by experimental bearing fault signal. The experimental system of a bearing test rig is shown in Fig. 5. The three-phase asynchronous motor whose type is YVF2-90L-4 has variable frequency and variable speed. Different speed conditions are simulated by tuning the motor speed by a frequency converter. Radial loading devices simulate radial loads in real operating conditions and the load is measured by the dynamometer. The planetary reducer is used to reduce the output speed and further meet the low-speed operation of magnetic powder brake. The type of magnetic powder brake is FZ-A-100. The input current of the magnetic powder brake is adjusted by the magnetic powder brake controller and it is used to change the output torque. In this case, we use it to simulate the torques in actual operating conditions. The fault bearing with the type of N306E is installed in the bearing seat at the end of the spindle. The experimental signal is collected by piezoelectric acceleration sensor with the type of 1A206E, which locates the bearing seat. Then, the collected signal is transmitted to a computer by acquisition card with the type of NI9234. Finally, we realise the data reading by the Labview software and DAQmx driver in a laptop. We do the fault diagnosis of the scratch fault in the outer ring here. The depth and width of the penetration scratch are 1.2 and $0.5 \mathrm{~mm}$, respectively. In the experiment, the rotational speed is $1421 \mathrm{rpm}$. The braking torque is $30 \mathrm{Nm}(1.2 \mathrm{~A})$ and the radial force is $0 \mathrm{~N}$. According to (10) and the structure size of 

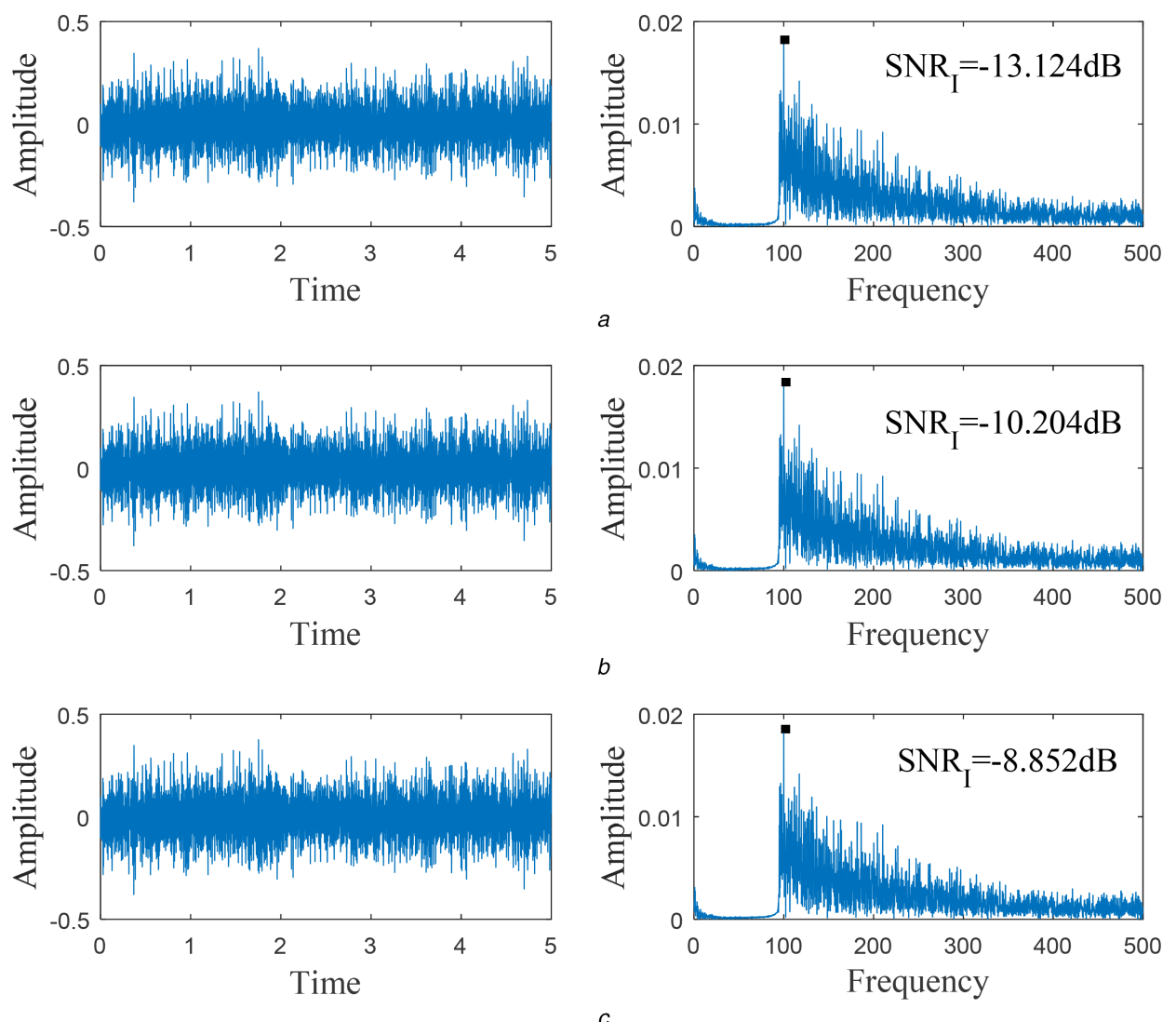

Fig. 3 Time-domain waveforms and frequency spectrums of the noisy signal after adaptive SR with the new index ISNR

(a) $l=5$ : Optimal output with $a=2.374$ and $b=1560.739$, (b) $l=10$ : Optimal output with $a=0.0638$ and $b=1312.799$, (c) $l=15$ : Optimal output with $a=1.377$ and $b=938.445$

Table 1 Values of the classic SNR and the new ISNR before and after adaptive SR, $f_{\mathrm{t}}=100 \mathrm{~Hz}, \Delta f_{\mathrm{t}}=0.2 \mathrm{~Hz}$

\begin{tabular}{lcccc}
\hline & SNR, dB & ISNR, dB & ISNR, dB & ISNR, dB \\
& $I=0$ & $I=5$ & $I=10$ & $I=15$ \\
\hline Before SR & -22.491 & -19.098 & -16.09 & -14.808 \\
After SR & -16.449 & -13.124 & -10.204 & -8.852 \\
\hline
\end{tabular}

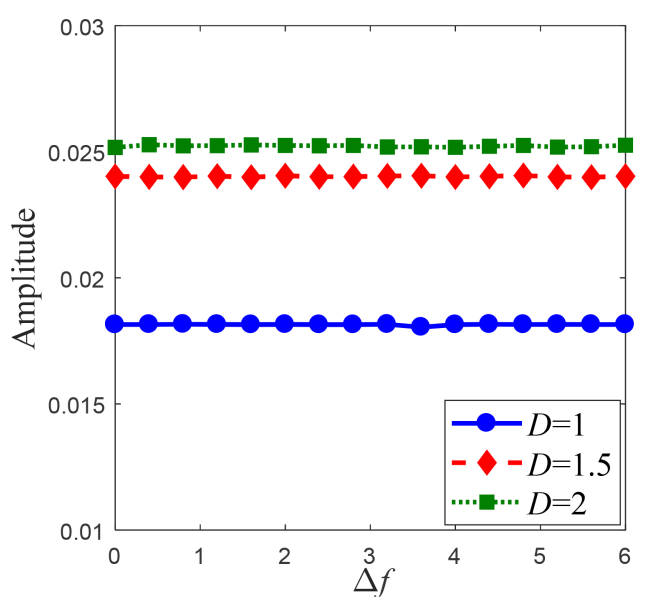

Fig. 4 Relationship between the amplitude of the fault frequency and bandwidth after adaptive $S R$

$\mathrm{N} 306 \mathrm{E}$, the theoretical fault frequency of bearing outer ring is $105.208 \mathrm{~Hz}$

$$
f_{\mathrm{t}}=\frac{Z}{2} \frac{N}{60}\left(1-\frac{D_{b}}{D_{c}} \cos \alpha\right)
$$

where $Z$ represents the number of rolling elements and $N$ stands for rotational speed. $D_{b}$ and $D_{c}$ symbolise the diameter of rolling element and pitch diameter of bearing, respectively. $\alpha$ is the contact angle.

In the analysis of the experimental signal, the sampling frequency is set as 12,800 and the number of sampling points is set as 64,000 . The real fault frequency of the fault bearing is $105 \mathrm{~Hz}$. In the calculations of SNR and ISNR, $M$ is set as 500 . The serial numbers $k_{\mathrm{t}}$ and $k_{\mathrm{r}}$ corresponding to the theoretical fault frequency $f_{\mathrm{t}}$ and accurate fault frequency $f_{\mathrm{r}}$ are 527 and 526, respectively. The passband frequency and stopband frequency of the high-pass filter are set as 100 and $95 \mathrm{~Hz}$, respectively. The scale of time is set as 1000 in general scale transformation. The system parameters $a$ and $b$ in adaptive SR are obtained by the random particle swarm optimisation algorithm. Fig. $6 a$ shows the time-domain waveform and frequency spectrum of the noisy signal under the high-pass filter and before adaptive SR. From Fig. $6 a$, we find the fault frequency is hard to be found as it is submerged in the noise.

First, the noisy signal is processed by the adaptive SR with classic index SNR. The time-domain waveform and frequency spectrum of the noisy signal after adaptive SR is shown in Fig. $6 b$. In Fig. $6 b$, the accurate fault frequency can be clearly identified in the frequency spectrum and the effect of adaptive SR with the classic index is satisfactory.

Then, the new index ISNR is used to process the noisy experimental signal in adaptive SR and three ranges are selected to demonstrate the efficiency of this new index. As the theoretical fault frequency is about $105.2 \mathrm{~Hz}$, three ranges are set as 104.2 106.2, 103.2-107.2 and 102.2-108.2 Hz, respectively. The related values of $l$ are set as 5, 10 and 15, respectively. Fig. 7 shows the time-domain waveforms and frequency spectrums about these three situations. Table 2 shows the values of the classic SNR and the new 


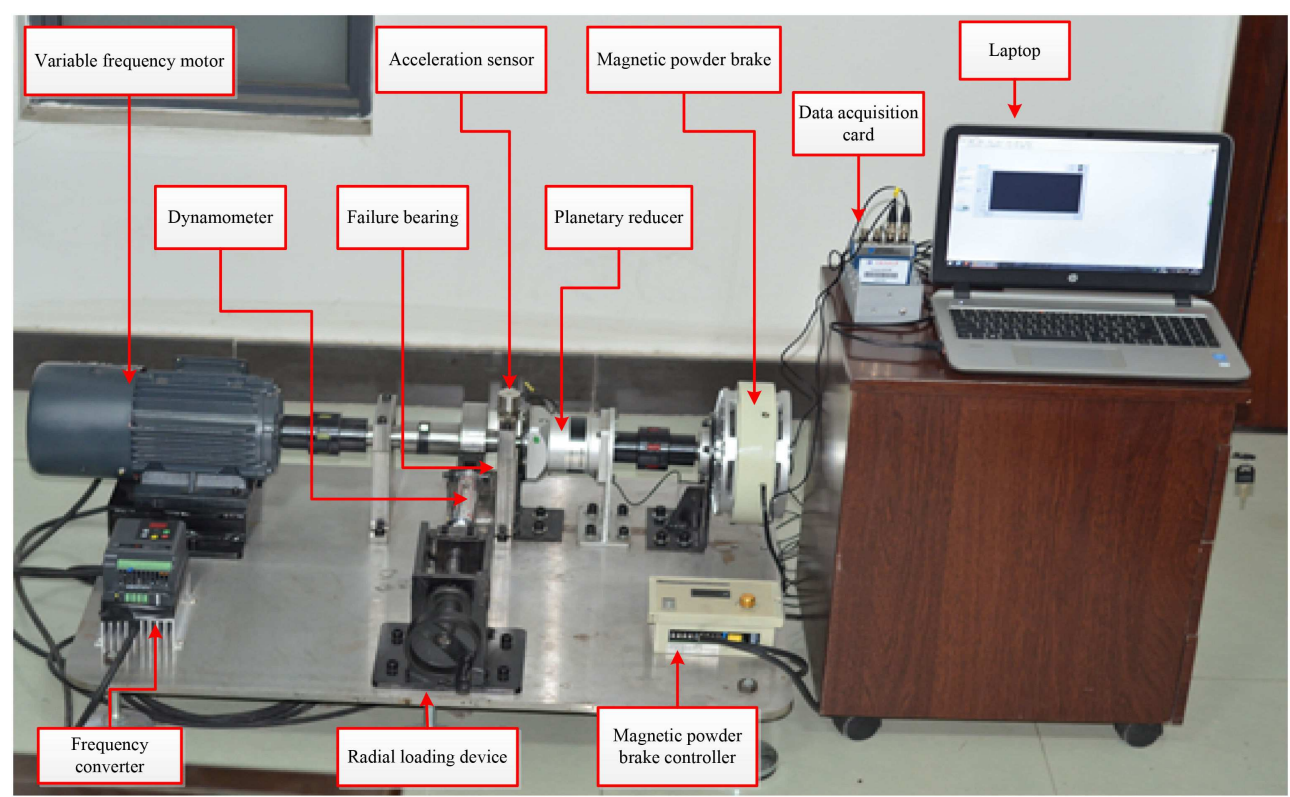

Fig. 5 Experiment system of the bearing test rig
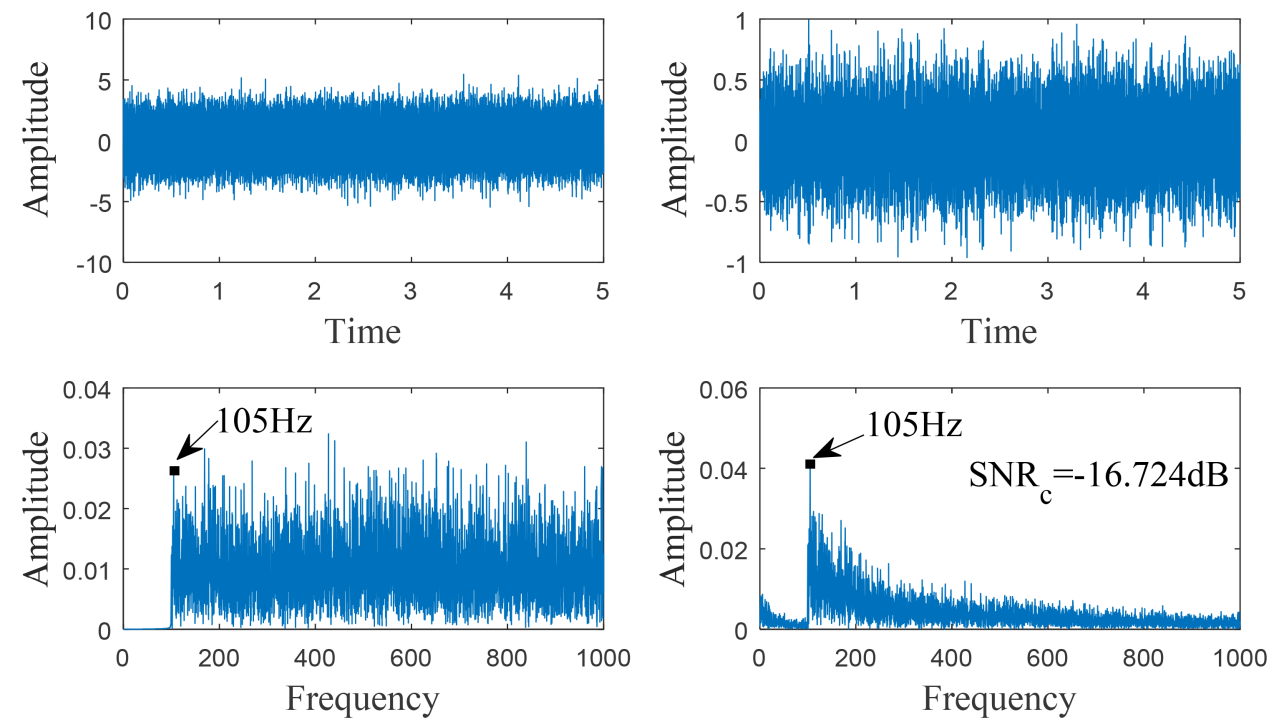

$b$

Fig. 6 Time-domain waveforms and frequency spectrums of the noisy signal before adaptive SR and after adaptive SR with the classic index SNR (a) Time-domain waveform and frequency spectrum of the noisy signal before adaptive SR, (b) Time-domain waveform and frequency spectrum of the noisy signal after adaptive SR with the classic index SNR. Optimal output with $a=51.869$ and $b=747.836$

ISNR before and after adaptive SR. From Fig. 7 and Table 2, we find the accurate fault frequency can be easily recognised and the values of ISNR are improved greatly after adaptive SR. Thus, the results which are processed by the adaptive SR with ISNR are satisfactory such as the classic SNR.

For purpose of proving the new index ISNR is suitable for a small range of fault frequency changes in different noise intensities such as simulation signal, Fig. 8 reveals the relationship between the amplitude of the real fault frequency and bandwidth after adaptive SR. In Fig. 8, the black points represent the amplitude of the real fault frequency in different noise intensities when the adaptive SR with classic index SNR is used. When $\Delta f$ is not equal to zero, the green points, red points and blue points symbolise the amplitude of the real fault frequency in different noise intensities by new index ISNR. Besides the colour points in the ordinate, the values of these colour points have little differences at the same noise intensity and have the same good effect as the SNR method. This result is the same as the situation in simulation signal. However, the green point, red point and blue point in the ordinate are the amplitude of real frequency when the ISNR is calculated with $l=0$. In other words, they are obtained when the SNR is calculated at theoretical fault frequency. However, the correct definition of SNR is calculated at the real fault frequency. So these points are not calculated by the correct definition of SNR or ISNR. For example, when the signal is processed by ISNR with $\Delta f=0$ at the noise intensity of 2 , this point has larger fluctuation compared with other points at the same noise intensity and shows a negative effect compared with the result of SNR. Therefore, the accurate fault frequency is always observed obviously when we look for it with the correct ISNR in the range of the neighbourhood of the theoretical fault frequency.

To verify the effectiveness of the ISNR in identifying the unknown frequency of failures, we give a detection case with real unknown characteristic frequency and the type of this fault and the value of accurate fault frequency are unknown. However, it is well known that rolling bearing has four typical faults which are outer ring fault, inner ring fault, rolling ball fault and cage fault, respectively. Therefore, we can calculate theoretical fault frequencies of these four typical faults in the rotational speed of $1425 \mathrm{rpm}$, which is shown by the speedometer. By the calculation formula of theoretical fault frequencies, we get the theoretical fault frequencies of the outer ring, inner ring, rolling ball and cage are 

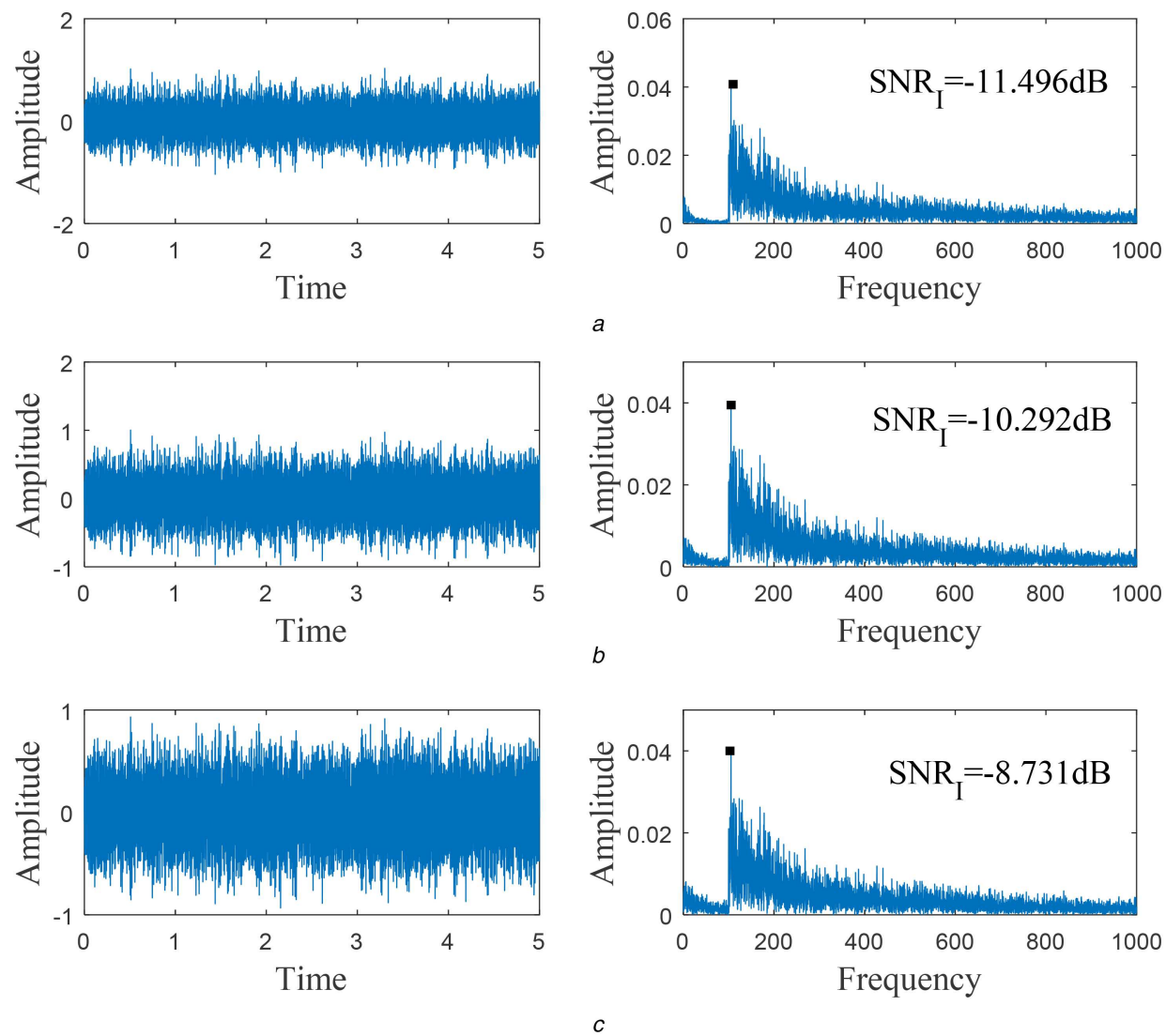

Fig. 7 Time-domain waveforms and frequency spectrums of the noisy signal after adaptive SR with the new index ISNR

(a) $l=5$ : Optimal output with $a=3.997$ and $b=217.926$, (b) $l=10$ : Optimal output with $a=21.366$ and $b=622.882$, (c) $l=15$ : Optimal output with $a=66.437$ and $b=1184.316$

Table 2 Values of the classic SNR and the new ISNR before and after adaptive SR, $f_{\mathrm{t}}=105.2 \mathrm{~Hz}, \Delta f_{\mathrm{t}}=0.2 \mathrm{~Hz}$

\begin{tabular}{lcccc}
\hline & SNR, dB & ISNR, dB & ISNR, dB & ISNR, dB \\
& $I=0$ & $I=5$ & $I=10$ & $I=15$ \\
\hline before SR & -19.529 & -14.373 & -13.18 & -11.507 \\
after SR & -16.724 & -11.496 & -10.292 & -8.731 \\
\hline
\end{tabular}

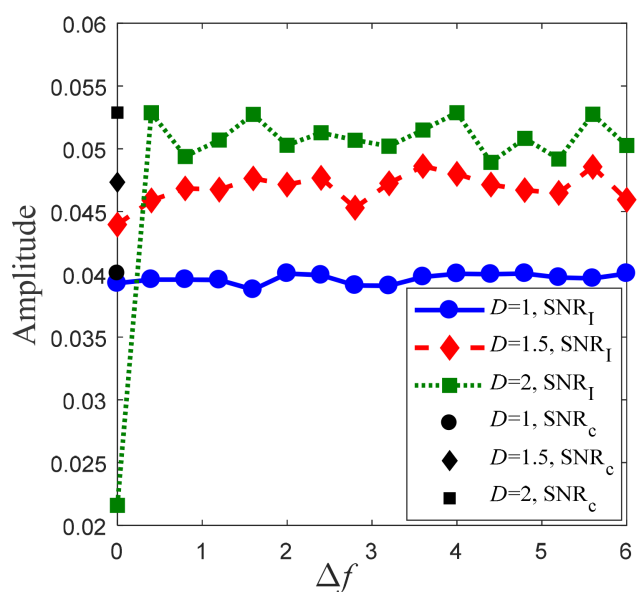

Fig. 8 Relationship between the amplitude of the real fault frequency and bandwidth after adaptive SR

105.5, 171.8, 119 and $9.6 \mathrm{~Hz}$, respectively. Fig. 9 demonstrates the results of this proposed method with the new ISNR, which is calculated by the above four theoretical fault frequencies. Fig. $9 a$ is obtained by the ISNR which is calculated at $105.5 \mathrm{~Hz}$ with $l=10$. As we know, there is a little error between the accuracy fault frequency and the theoretical fault frequency. However, the highest point in Fig. $9 a$ is $117.8 \mathrm{~Hz}$ which has great errors with $105.5 \mathrm{~Hz}$ and interference components around it also exists. Therefore, it is not an outer ring fault and the accuracy fault frequency of rolling ball is shown. Fig. $9 b$ is got by the ISNR which is calculated at
$171.8 \mathrm{~Hz}$ with $l=10$. This failure result proves it is not an inner ring fault. Fig. $9 c$ is received by the ISNR which is calculated at $119 \mathrm{~Hz}$ with $l=10$. The result is satisfactory and the accurate fault frequency is $117.8 \mathrm{~Hz}$, which is close to the theoretical fault frequency. Fig. $9 d$ is got by the ISNR which is calculated at $9.6 \mathrm{~Hz}$ with $l=10$ and the result is unsatisfactory. Therefore, Figs. $9 a, b$ and $d$ demonstrate that there are no outer ring fault, inner ring fault and cage fault of this unknown signal. Figs. $9 a$ and $c$ prove this unknown signal has rolling ball fault and the accurate fault frequency is $117.8 \mathrm{~Hz}$.

In practical engineering occasions, we do not know the accurate fault frequency first. In these conditions, it is meaningless to process signals by the classic SNR with theoretical fault frequency and the results might also be unsatisfactory. However, the proposed ISNR includes the accuracy fault frequency because it searches the accuracy fault frequency within the theoretical frequency range. Moreover, the ISNR has superior advantages when the accuracy fault frequency is always changing. For example, the changes of speed and load in working condition always lead to the variety of the fault frequency. In view of this situation, the new index ISNR which always includes the accuracy fault frequency within a certain range is suitable for the situation of variable frequency. In summary, the adaptive SR with ISNR has specific advantages in signal processing and fault diagnosis.

\section{Conclusion}

Herein, we mainly propose a new index ISNR in adaptive SR to extract the weak feature information in the noise background. 


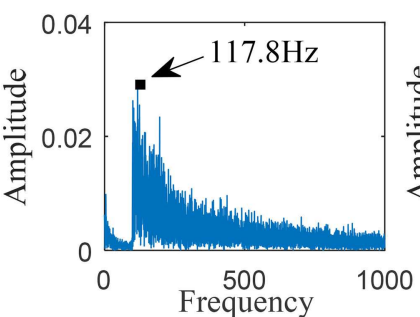

a

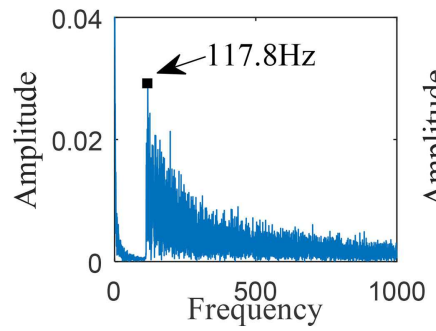

$c$

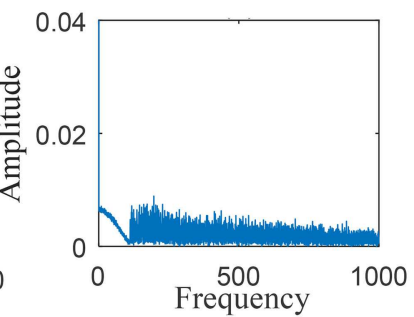

$b$

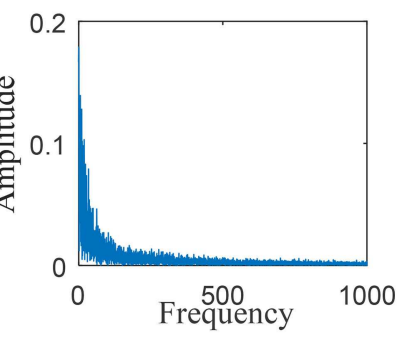

$d$
Fig. 9 Frequency spectrums of the unknown signal after adaptive SR with the new index ISNR, which is calculated by the theoretical fault frequencies of the outer ring, inner ring, rolling ball and cage, respectively

(a) ISNR is calculated by the theoretical fault frequency of outer ring with $l=10$ : optimal output with $a=7.904$ and $b=612.628$, (b) ISNR is calculated by the theoretical fault frequency of inner ring with $l=10$ : optimal output with $a=1435.707$ and $b=371.198$, (c) ISNR is calculated by the theoretical fault frequency of rolling ball with $l=10$ : optimal output with $a=20.788$ and $b=46.679$, (d) ISNR is calculated by the theoretical fault frequency of cage with $l=10$ : optimal output with $a=760.316$ and $b=1369.539$

Compared to the classic index SNR, it has the following advantages:

I. When we use the new ISNR index in adaptive SR, it does not need to know the accurate characteristic frequency in prior different from the classic index SNR. The accurate characteristic frequency near the theoretical frequency is easy to be found by the adaptive SR with ISNR. Therefore, the new index ISNR is more specifically suited to the actual situation.

II. Both the simulation signal and experimental bearing fault signal are used to verify the adaptive SR with ISNR, and they have the same good performance.

III. The proposed new index ISNR in adaptive SR has strong robustness and it is also suitable for another case such as the characteristic frequency oscillates in a small scope. Consequently, this method might have practical application values in signal processing and vibration fault characteristic information extraction.

The proposed method is suitable for fault frequency changes in a small scope, which is caused by the unstable working condition. When the bearing operates under a time-varying rotational speed condition and the variable speed is changing in a larger range, this proposed method is not applicable. This problem is also our future research issue.

\section{Acknowledgments}

We acknowledge financial supports by the National Natural Science Foundation of China (Grant no. 11672325) and the Topnotch Academic Programs Project of Jiangsu Higher Education Institutions and the Priority Academic Program Development of Jiangsu Higher Education Institutions.

\section{References}

[1] Benzi, R., Sutera, A., Vulpiana, A.: 'The mechanism of stochastic resonance', J. Phys. A, Math. Gen., 1981, 14, (11), pp. 453-457

[2] Singh, A., Parey, A.: 'Gearbox fault diagnosis under fluctuating load conditions with independent angular re-sampling technique, continuous wavelet transform and multilayer perceptron neural network', IET Sci. Meas. Technol., 2017, 11, (2), pp. 220-225

[3] Wang, J., He, Q.B.: 'Wavelet packet envelope manifold for fault diagnosis of rolling element bearings', IEEE Trans. Instrum. Meas., 2016, 65, (11), pp. 2515-2526

[4] Hui, K.H., Ooi, C.S., Lim, M.H., et al.: 'A hybrid artificial neural network with Dempster-Shafer theory for automated bearing fault diagnosis', $J$. Vibroeng., 2016, 18, (7), pp. 4409-4418

[5] Yuan, H.Y., Wang, X.Y., Sun, X., et al.: 'Compressive sensing-based feature extraction for bearing fault diagnosis using a heuristic neural network', Meas. Sci. Technol., 2017, 28, (6), p. 065018

[6] Van, M., Kang, H.J.: 'Bearing-fault diagnosis using non-local means algorithm and empirical mode decomposition-based feature extraction and two-stage feature selection', IET Sci. Meas. Technol., 2015, 9, (6), pp. 671680

[7] Xiong, Q., Xu, Y.H., Peng, Y.Q., et al.: 'Low-speed rolling bearing fault diagnosis based on EMD denoising and parameter estimate with alpha stable distribution', J. Mech. Sci. Technol., 2017, 31, (4), pp. 1587-1601

[8] Shi, P.M., An, S.J., Li, P., et al.: 'Signal feature extraction based on cascaded multistable stochastic resonance denoising and EMD method', Measurement, 2016, 90, (1), pp. 318-328

[9] Li, J.M., Chen, X.F., He, Z.J.: 'Adaptive stochastic resonance method for impact signal detection based on sliding window', Mech. Syst. Signal Process., 2013, 36, (2), pp. 240-255

[10] Qin, Y., Tao, Y., He, Y., et al.: 'Adaptive bistable stochastic resonance and its application in mechanical fault feature extraction', J. Sound Vib., 2014, 333, (26), pp. 7386-7400

[11] Lei, Y.G., Han, D., Lin, J., et al.: 'Planetary gearbox fault diagnosis using an adaptive stochastic resonance method', Mech. Syst. Signal Process., 2013, 38, (1), pp. 113-124

[12] Liu, X.L., Yang, J. H., Liu, H.G., et al:: 'Optimizing the adaptive stochastic resonance and its application in fault diagnosis', Fluct. Noise Lett., 2015, 14, (4), p. 1550038

[13] Chen, X.H., Cheng, G., Shan, X.L., et al.: 'Research of weak fault feature information extraction of planetary gear based on ensemble empirical mode decomposition and adaptive stochastic resonance', Measurement, 2015, 73, (1), pp. 55-67

[14] Marini, F., Walczak, B.: 'Particle swarm optimization (PSO). A tutorial', Chemometr. Intell. Lab., 2015, 149, (1), pp. 153-165

[15] Guedria, N.B.: 'Improved accelerated PSO algorithm for mechanical engineering optimization problems', Appl. Soft. Comput., 2016, 40, (40), pp. 455-467

[16] Zhang, J.L., Huang, D.W., Yang, J.H., et al.: 'Realizing the empirical mode decomposition by the adaptive stochastic resonance in a new periodical model and its application in bearing fault diagnosis', J. Mech. Sci. Technol., 2017, 31, (10), pp. 4599-4610

[17] Ichiki, A., Tadokoro, Y.: 'Signal-to-noise ratio improvement by stochastic resonance in moments in non-dynamical systems with multiple states', Phys. Lett. A, 2013, 377, (3-4), pp. 185-188

[18] Tan, J.Y., Chen, X.F., Wang, J.Y., et al.: 'Study of frequency-shifted and rescaling stochastic resonance and its application to fault diagnosis', Mech Syst. Signal Process., 2009, 23, (3), pp. 811-822

[19] Leng, Y.G., Leng, Y.S., Wang, T.Y., et al.: 'Numerical analysis and engineering application of large parameter stochastic resonance', J. Sound Vib., 2006, 292, (3), pp. 788-801

[20] Leng, Y.G., Wang, T.Y., Guo, Y., et al.: 'Engineering signal processing based on bistable stochastic resonance', Mech. Syst. Signal Process., 2007, 21, (1), pp. $138-150$

[21] Dai, D., He, Q.: 'Multiscale noise tuning stochastic resonance enhances weak signal detection in a circuitry system', Meas. Sci. Technol., 2012, 23, (11), p 115001

[22] He, Q., Wang, J.: 'Effects of multiscale noise tuning on stochastic resonance for weak signal detection', Digit. Signal Process., 2012, 22, (4), pp. 614-621

[23] Hang, D.W., Yang, J.H., Zhang, J.L., et al.: 'An improved adaptive stochastic resonance method for improving the efficiency of bearing faults diagnosis', preprint, 2017, 10.1177/0954406217719924

[24] Mcnamara, B., Wiesenfeld, K.: 'Theory of stochastic resonance', Phys. Rev $A, 1989,39,(9)$, pp. $4854-4869$

[25] Gammaitoni, L., Hänggi, P., Jung, P., et al.: 'Stochastic resonance', Rev. Mod. Phys., 1998, 70, (1), pp. 223-287

[26] Lu, S.L., He, Q.B., Zhang, H.B., et al. 'Enhanced rotating machine fault diagnosis based on time-delayed feedback stochastic resonance', J. Vib. Acoust., 2015, 137, (5), p. 051008

[27] Lu, S.L., He, Q.B., Kong, F.R.: 'Effects of underdamped step-varying secondorder stochastic resonance for weak signal detection', Digit. Signal Process., 2015, 36, (1), pp. 93-103 